\title{
SAFER Inspection of Space Shuttle Thermal Protection System
}

\author{
Zebulon C. Scoville* and Sudhakar Rajula ${ }^{\dagger}$ \\ NASA Johnson Space Center, Houston, TX, 77058
}

\begin{abstract}
In the aftermath of the space shuttle Columbia accident, it quickly became clear that new methods would need to be developed that would provide the capability to inspect and repair the shuttle's thermal protection system (TPS). A boom extension to the Remote Manipulator System (RMS) with a laser topography sensor package was identified as the primary means for measuring the damage depth in acreage tile as well as scanning Reinforced CarbonCarbon (RCC) surfaces. However, concern over the system's fault tolerance made it prudent to investigate alternate means of acquiring close range photographs and contour depth measurements in the event of a failure. One method that was identified early was to use the Simplified Aid For EVA Rescue (SAFER) propulsion system to allow EVA access to damaged areas of concern. Several issues were identified as potential hazards to SAFER use for this operation. First, the ability of an astronaut to maintain controlled flight depends upon efficient technique and hardware reliability. If either of these is insufficient during flight operations, a safety tether must be used to rescue the crewmember. This operation can jeopardize the integrity of the Extra-vehicular Mobility Unit (EMU) or delicate TPS materials. Controls were developed to prevent the likelihood of requiring a tether rescue, and procedures were written to maximize the chances for success if it cannot be avoided. Crewmember ability to manage tether cable tension during nominal flight also had to be evaluated to ensure it would not negatively affect propellant consumption. Second, although propellant consumption, flight control, orbital dynamics, and flight complexity can all be accurately evaluated in Virtual Reality (VR) Laboratory at Johnson Space Center, there are some shortcomings. As a crewmember's hand is extended to simulate measurement of tile damage, it will pass through the vehicle without resistance. In reality, this force will push the crewmember away from the vehicle, and could induce a moment which, if strong enough, could saturate the attitude control system in SAFER. This raises the concern that additional propellant will be consumed to maintain controlled flight. To account for this, the fidelity of the Virtual Reality simulation was improved to include the effect of crewmember contact with the vehicle during SAFER flight. In addition, while participating in VR simulations, the subject is in shirtsleeves and sits in a chair. This does not provide a flight-like representation of body position awareness. To prevent inadvertent contact with tile or RCC, other facilities were utilized to establish crew preferences for body attitude and tool configuration. Finally, a study was performed to determine if attitude constraints are needed for the Space Shuttle and International Space Station to reduce SAFER flight difficulty.
\end{abstract}

\section{Introduction}

$\mathrm{O}$ February $1^{\text {st }}, 2003$ one Israeli and six American astronauts died when the space shuttle Columbia disintegrated during reentry. In August the same year, the Columbia Accident Investigation Board (CAIB) issued a report that detailed the cause of the disaster. Insulating foam separated from the external tank and impacted the leading edge of the left wing 81.7 seconds after launch. The resultant damage that was caused to the Reinforced Carbon-Carbon $(\mathrm{RCC})^{\ddagger}$ allowed hot gas and plasma to burn through the left wing during reentry. Loss of attitude control and overwhelming stress on the vehicle caused Columbia and her crew to break up over East Texas.

\footnotetext{
*EVA Flight Controller/Crew Trainer, EVA Systems Group, 2101 NASA Road One, DX3

† Manager, Anthropometry \& Biomechanics Facility, Habitability \& Human Factors Office, 2101 NASA Rd 1, SF3

$\ddagger$ RCC is a composite structure that provides thermal protection for the space shuttle in areas that experience the highest heat loads during reentry. It is located on the vehicle nose and wing leading edges.
}

1 
Included in the CAIB report was a number of recommendations that the board suggested be implemented prior to returning the space shuttle to flight. One such recommendation was that NASA "develop[s] a practicable capability to inspect and affect emergency repairs to the widest possible range of damage to the Thermal Protection System, including both tile and Reinforced Carbon-Carbon." In fulfillment of this recommendation, existing inspection methods are being improved and a suite of new techniques are being developed. High-speed video and precision radar will be used from the ground. To complement this, NASA's WB57 high altitude aircraft will record video of the shuttle during ascent. Onboard the vehicle, cameras mounted in the external tank umbilical well ${ }^{\S}$ will be upgraded to provide imagery downlink capability for ground analysis during the mission. Crew handheld video cameras will film the external tank after separation to try to detect areas of missing foam. Accelerometers are being added to all RCC panels in the wings. If an object strikes an RCC panel, the sensors will record an acceleration spike significantly greater than the nominal levels seen during launch. While all of these tools help to characterize the environment around the space shuttle, the data are indirect indicators of risk. None of these means provide a direct indication of the severity of damage that the Orbiter has sustained during launch. These assets can supply the vital information that debris was present, its mass properties can be determined, and at best it can indicate that an object hit the vehicle. While analytical models can use this information to predict damage, there is always uncertainty in the source data and limitations to the applicability of the model itself. The value gained from a direct on-orbit inspection of the thermal protection system cannot be understated. The best way to assess the health of the critical materials after launch is to look at it. When the size, shape, depth and location of damage is known the capability to accurately predict the survivability of reentry improves.

One of the largest obstacles faced when attempting to inspect the space shuttle thermal protection system is that the majority of the surfaces are obstructed from the view of the crew cabin and robotic arm. In addition, traditional EVA handholds and translation aids do not exist near TPS surfaces making it impossible to send a person to the location using existing means. Because of this, new hardware and procedures are being developed to support the shuttle's return to flight. The most radical change is the addition of the Orbiter Boom Sensor System (OBSS). This hardware consists of a sensor package mounted on the end of a long pole. When attached to the existing robotic arm, the OBSS will have visual access to otherwise obscured locations. The sensor system will provide real-time, twodimensional video. In addition, the data from a scanning laser can be used to map the surface such that threedimensional contours of any damage can be recreated on the ground ${ }^{* *}$. Unfortunately, performing scans with the OBSS is a major impact to the mission timeline. Therefore, it is impractical to examine the entire vehicle with OBSS. Because the greatest aerodynamic and thermal demands are placed on RCC, the inspection requires the highest resolution that only the OBSS can provide. The robotic arm will grapple the boom to perform a full day inspection of the RCC on both wings and the nose cap. ${ }^{\text {tt }}$

The short duration of a space shuttle mission does not make an OBSS scan of all TPS surfaces realistic. Effectively the majority of on orbit time would be spent scanning tile and all mission goals and utility would be lost if this were attempted. Fortunately the Orbiter can tolerate larger damage to the vast majority of tiles on the lower surface of the vehicle compared to RCC. The area of black tile that comprise the shuttle's lower surface is referred to as acreage tile. This greater damage tolerance permits the resolution requirements for imagery of acreage tile to be met with a digital camera held by the International Space Station (ISS) crew. As the shuttle approaches ISS, it will present its underside to the space station crew during what is known as the Rendezvous Pitch Maneuver (RPM). At a range of approximately 600 feet, the acreage tile will be exposed for 93 seconds of photography.

To close out the on-orbit inspection, the preexisting end effector camera on the space shuttle's robotic arm will be used to capture close-up video of the crew cabin. From the aft flightdeck, a handheld digital camera will photograph the vertical stabilizer and Orbital Maneuvering System (OMS) tiles. If damage is observed here, or

$\S$ During ascent, two doors on the underside of the space shuttle are open to allow umbilicals to supply liquid hydrogen and oxygen from the external tank to space shuttle main engines. Imagery from this location is desired because ice debris is often generated from the cryogenically cooled lines and it provides views of the external tank.

${ }^{* *}$ There are several benefits to 3-D data as opposed to 2-D images. The amount of material remaining in a location can aid in predicting if plasma will burn through to the aluminum structure. It may also be possible to predict if the perturbations to the airflow are great enough to cause a transition from laminar to turbulent flow. Early transition to turbulent flow can cause large increases in downstream heating of the Orbiter.

it At time of print, a goal of the first two shuttle missions after the Columbia accident is to better characterize the nominal signatures of the wing leading edge accelerometers and to demonstrate that external tank redesigns have sufficiently reduced debris shedding. If this is successful, subsequent flights will not map the RCC surfaces with OBSS. Rather a point inspection would be performed in areas of concern indicated by the accelerometers or other imagery assets. 
during the Rendezvous Pitch Maneuver, the two-dimensional imagery may be insufficient to determine if damage is survivable. If suspicious damage brings the safety of the crew into question, time has been reserved to perform an additional OBSS point inspection at these locations. This would provide the three dimensional information required for TPS experts to make the most informed reentry decision.

When all the inspection plan pieces described above come together, the recommendation of the CAIB report is satisfied; all critical TPS components can be viewed with adequate resolution. If history can be any guide, we must recognize that space flight hardware does not always operate as planned on its maiden voyage. Inevitably, NASA is forced to prepare for the possibility that hardware's unexpected 'features' may prevent it from delivering the desired results. There are OBSS and robotic arm components that are zero fault tolerant; a single failure in these systems has the potential to deny inspection capability with the boom. In some cases the sensor may be inoperable, while other failures may completely prevent removal of the boom from its launch location. Although it is less, the RPM photography of the acreage tile is not without risk either. The sun and Earth must be in proper alignment to obtain the necessary lighting conditions. The predicted window of opportunity to capture all images is 93 seconds. Because these pictures are taken with a handheld digital camera, it is possible that the crew will not obtain photographic coverage of the entire surface. Regardless of the particular chain of events and anomalies that come to pass, many circumstances necessitate an alternate method of inspection.

When a backup system is chosen, it must function independently of the failures that would require its use. Therefore, a prerequisite of the contingency inspection method is that it operates without the OBSS. Any robotics operations must be able to be performed using either space station's (SSRMS) or shuttle's (SRMS) robotic arm. Considering these limitations, it was decided that EVA techniques should be developed for sending a person to examine the thermal protection system. At fist glance, it would seem that utilizing the same methods that allow a crewmember access to repair damage could also place them in close proximity for detailed inspections. In fact, this is not the case. If damage is located outside the reach of either robotic arm, the instability of the boom as a work platform may make repair impossible from this location. If this is the case, the space shuttle would be required to perform the Orbiter Repair Maneuver (ORM). This is a three-day operation in which the space shuttle undocks from ISS and uses the SRMS to present its underside to EVA crewmembers on the SSRMS. The hazards associated with this procedure place both the vehicles at risk and a tremendous amount of time is required for execution. Consequently, different ways are needed for EVA inspection access than those used for repair.

The backup inspection method used is very dependent upon the damage location. In many circumstances, the simplest approach is the best. If the damage to be inspected can be reached by a crewmember on the end of a robotic arm, then this is clearly desired. A foot restraint on the robotic arm is stable and secure. Additionally, there is extensive flight experience with this configuration. Moreover, several tools can be stacked on the end of the arm to extend the crewmember's reach. In addition, it is possible that the backup inspection is only required because the OBSS sensor package failed. The boom itself might still be available. If so, consideration will be given to an EVA inspection based off the boom's end.

There are scenarios that preclude EVA inspection from a robotic platform. Quite simply, the boom could be unavailable and the damage beyond the range of other robotic and EVA tools. Similarly, on a flight specific basis, there may be a prohibitive amount of overhead involved in relocating space station hardware for use in this task. Even if the boom was available, dynamic analysis may indicate that flexure and instability with a 680-pound crewmember on the boom could risk further damage through secondary impacts to the shuttle. A new method must be available to extend the reach of EVA crewmembers.

During the conceptual stages of the International Space Station, it was recognized that there would be no way to fly after, and retrieve an EVA astronaut who had suffered a failure of their safety tether and become separated from structure. During stand-alone Space Shuttle missions, like those to repair the Hubble telescope, the Orbiter could chase down a stranded crewmember. This is not possible for EVAs conducted on ISS. The solution was to develop a self-contained propulsion system that could be attached to the space suit. In the event that an astronaut separates from station, they could activate this jet pack and rescue themselves by flying back to the vehicle. The propulsion system that was built is called the Simplified Aid For EVA Rescue (SAFER). It is now worn on every EVA conducted from the space station. What if it were used to fly away from solid structure rather than exclusively as a rescue tool to return to it? If this is done, EVA crewmembers could fly to the lower surface of the space shuttle to conduct a detailed inspection and then return safely. Herein lies a backup method to satisfy the on-orbit inspection recommendation of the CAIB report. 


\section{SAFER System Description}

In order to understand the challenges facing the use of SAFER as an Orbiter inspection tool, it is first necessary to become familiar with the existing system design. SAFER is a cold gas system that provides six degrees-offreedom in translation and rotation. This allows a pilot to fly up and down, left and right, forward and backward. Roll, pitch and yaw rotations are also possible. SAFER attaches to the life support backpack of the Extravehicular Mobility Unit (EMU), or spacesuit. By sliding a lever up, a stowage tray containing the SAFER Hand Control Module ( $\mathrm{HCM}$ ) is deployed and swings to the front of the crewmember. Grasping the HCM with two hands, the astronaut can control flight using a four degree-of-freedom joystick mounted on its right side. Thrust is achieved by flowing pressurized gaseous nitrogen through any of the twenty-four jet nozzles on SAFER.

The nitrogen propellant is stored in a tank that is pressurized to 8000 psi. This quantity of gas is capable of accelerating the 680 pound combined weight of a crewmember and EMU to a speed of 10 feet per second. SAFER also has the capability to stabilize the crewmember's attitude. This is accomplished with a three-axis fiber optic gyroscope that measures rotational velocities in roll, pitch, and yaw. Control software uses the signals from the gyroscopes and commands particular thrusters to fire and neutralize rotation. This functionality is called Automatic Attitude Hold (AAH). Power is provided to the thruster

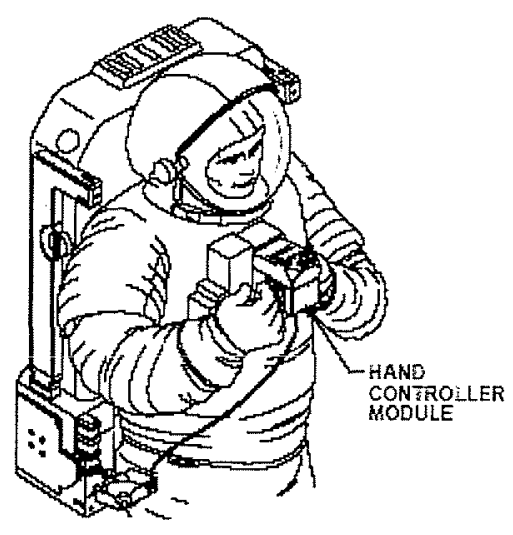

Figure 1. SAFER attached to EMU valves, avionics, and HCM from a dedicated battery. Power and propellant are limited resources on the SAFER. A small display on the HCM allows the crewmember to monitor the remaining quantity of these consumables.

When existing hardware is to be used in a role for which it was never intended, it is important that the performance requirements to which it was designed are compared to those that it will be expected to fulfill. If the demands of the new task cannot be met with the existing equipment, then hardware may need to be modified. In some cases however, the hardware has more capability than its original certification suggests. If this is the case, it is necessary to prove that new tasks actually are within the hardware's capability and the certification should be updated. SAFER rescue operations are significantly different from Orbiter inspection. Where rescue flights only last 13 minutes, inspection requires up to forty-five minutes of flight time. Another difference is that an astronaut using SAFER for rescue uses two hands to operate the hand controller; one hand manipulates the joystick while the other holds the HCM steady. Conversely, inspection requires that both a digital camera and depth gauge be used while operating the hand controller. This dictates that the HCM be rigidly secured in front of the crewmember to enable one-handed operation. A final subtle distinction between the two uses of SAFER relates to fault tolerance. EVA hardware is required to be single fault tolerant. This means that no single failure of hardware can jeopardize crew safety. However, hardware is not required to be safe in the event of any two failures. During an EVA, the spacesuits are always double tethered to the vehicle. Before a SAFER rescue is needed, two tether failures would have to occur. With this in mind, SAFER system redundancy was not required ${ }^{\ddagger \pm}$. This means that a single failure could render it useless. Alternatively, an inspection flight would be performed without any existing failures in EVA hardware. Essentially this means that crew safety would be dependent upon a device that may not operate after a single component failure. The existing safety tether is only 55 feet long, which is too short to be used as an additional level of protection for SAFER flights to remote damage locations. In order to regain the mandatory redundancy, a new 85-foot safety tether has been designed for SAFER inspection. However, tethered flight raises new safety concerns as will be discussed in the following section.

\section{Problem Description}

While considering the new requirements that vehicle inspection imposes on SAFER, several problems were identified that would require resolution before flight use of the hardware. The questions that raised the greatest concern are identified here.

The SAFER is a simplified design based upon the Manned Maneuvering Unit (MMU). The MMU was single fault tolerant, however, the redundancy came at the price of prohibitive size. In order for the SAFER to be reduced to a practical size for standard use, fault tolerance was sacrificed. 


\section{A. Risk of Safety Tether Rescue}

Adapting the application of existing SAFER hardware to satisfy a new mission requires the use of a safety tether during flight to retain the necessary fault tolerance. However, if controlled flight capability is lost and it becomes necessary to retrieve a stranded crewmember by retracting the tether, new risks are created. Probably the most disconcerting is the result of conserved angular momentum. Angular momentum can be expressed as:

$$
L=R \times M V
$$

In Eq. (1), the mass of the EMU, $M$, is constant. The distance between the SAFER pilot and the point at which their safety tether is attached to the robotic arm is written as $\mathrm{R}$. The linear velocity of the crewmember is expressed as V. Equation 1 can be simplified by replacing $V$ with the component of the velocity that is perpendicular to the vector $R$.

$$
L=R M V \sin \theta
$$

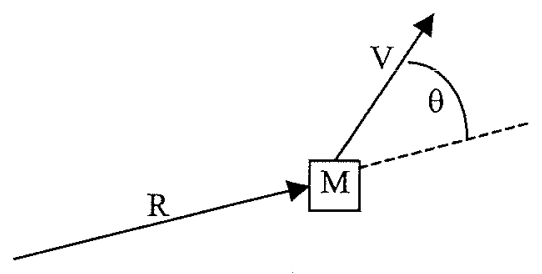

Theta is the angle between the $\mathrm{V}$ and $\mathrm{R}$. Because angular momentum is conserved, $\mathrm{L}$ at the time of SAFER failure $(t=0)$ must equal $L$ after the tether has been retracted $(t=1)$. Therefore, Eq. (2) can be written:

$$
R_{0} V_{0} \sin \theta_{0}=R_{1} V_{l} \sin \theta_{1}
$$

The implications of this are severe for SAFER rescue. A slowly moving crewmember at the end of an extended tether would be accelerated far beyond the EMU impact certification limits after being pulled toward the robotic arm. How can the safety tether be relied upon as a level of fault tolerance if its use could risk catastrophic damage to the spacesuit or Orbiter?

The danger of safety tether retrieval can be reduced two ways. As long as flight control can be maintained, the operation is not required and the hazard can be avoided. Second, if control is lost and a tether retrieval must be performed, every reasonable effort must be made to make the procedure as safe as possible. The fundamental causes of lost control are uncommanded thruster firings, failure of thrusters to fire when needed, loss of propellant, and inadequate piloting skill. Analysis, testing, and operational procedures are needed to keep this risk as low as possible. One of the most significant concerns surrounding SAFER use for inspection involves the longer duration flight. For a rescue scenario, the hardware was only designed and certified to operate for a minimum of thirteen minutes. During TPS inspection there may be multiple locations that need investigation. Each of these sites would require photographs, and depth measurements may also be needed. The necessary time to complete these tasks is significantly longer. The actual hardware capability had to be known in order to define the allowable flight duration before a system failure could cause loss of control. Each individual SAFER component was scrutinized to determine its operational life in the worst-case temperature extremes. Battery capacity was tested to ensure adequate power was available to support longer missions. This analysis revealed that the limiting component is the fiber-optic gyroscope used to stabilize the attitude. After forty-five minutes of powered operation, these components could overheat and fail. A further probabilistic risk assessment was conducted to quantify the risk of a random hardware failure within this forty-five minute window that would cause a loss of control.

In addition to hardware analysis, testing was needed to quantify the risk of human error. Ever since SAFER's beginning, a large variation in crewmember piloting skill has been observed. This is evidenced by the propellant consumption and the time required to perform a successful rescue during training sessions in Johnson Space Center's Virtual Reality (VR) Laboratory. During a rescue, $100 \%$ of the available propellant could be used to complete the mission. It was not necessary to preserve any gas for subsequent operations. For an inspection task the crewmember needs to fly away from the robotic arm, photograph and measure the damage depth, and then either proceed to a second damage location or return to the arm. It is necessary to set limits on remaining propellant and 
flight time. These bounds would be set such that if they were ever reached, adequate margin would remain for the crewmember to safely return to the robotic arm. How should these limits be set? Because rescue and inspection missions are so different, there was not any previous experience applicable for defining these limits. Furthermore, because crewmember capability varies, the limits must be sufficiently conservative such that any astronaut performing the task will be able to reach safety every time.

\section{B. Effects of Vehicle Contact Forces}

Ground based tests of flight hardware are often limited in realism because they lack both the vacuum and weightlessness of space. Because of this, the most realistic simulation of SAFER flight is achieved in a virtual reality laboratory. Only here can the performance characteristics of the jets and attitude control system be modeled. This also allows the crewmembers to see a visual image of ISS and the space shuttle. Unfortunately, the virtual environment is not without problems. Because the vehicles are only represented by graphical models, they provide no resistance during contact. This means that it is possible to fly through solid structures in the VR lab. The implications of this are significant for inspection flight studies. As previously discussed, it will be necessary to measure the damage depth. This will be accomplished during the SAFER inspection by spanning the damage with a contour gauge. The distance between the base of the tool and tile surface is then measured. A necessary condition for operation of the depth gauge is that it contacts the healthy tile surface on either side of the damage. In orbit, this contact will impart a reaction force into the EMU. However, in the VR lab the tool simply passes through the vehicle without affecting flight. In order to quantify accurately the amount of propellant needed for inspection, the reaction force cannot be neglected. The crewmember may need to expel additional gas to maintain their position in front of damage so that multiple measurements can be taken. In addition, if the tool is held far from the EMU's center of gravity during contact, it is unknown if the resultant moment could create a large angular displacement before the attitude control system could stop rotation. If this were to happen, considerable propellant would be consumed during recovery. In the worst case, crewmember disorientation could jeopardize safety. A new method needed to be developed for analyzing the effects of these reaction forces. This is complicated by human-in-the-loop damping actions of the person's arm that holds the tool.

\section{Body and Tool Position Awareness}

Many simple skills are often taken for granted before donning an EMU. Without looking, people are aware of the locations of their body parts. This becomes much more difficult in a spacesuit. The added volume of the spacesuit and accompanying tools, combined with a very restricted field of view, makes it difficult to know how much clearance exists between one's EMU and nearby objects. Although TPS materials can withstand some light contact, accidentally kicking tiles or RCC could certainly cause unwanted collateral damage. Is there a body position that keeps damage within measurement reach, but also provides adequate sensory cues to assure the crewmember that they are not at risk of unintentionally contacting the Orbiter surface with stray tools and suit parts?

\section{Safety Tether Slack Management}

Although a safety tether is needed in the event of a SAFER failure, during nominal flight operations the effects of the tether must be minimized. Any tension on the tether cable will impart an unbalanced pull on the EMU. This force will create unwanted motion unless it is counteracted by thruster firings. When this happens, nitrogen propellant is wasted. If left untended, the safety tether would continuously tug on the EMU because the safety tether reel is built with an internal torsion spring that automatically retracts loose cable. To minimize this effect, the second EVA crewmember will be positioned in a foot restraint on the end of the robotic arm. During inspection, this crewmember will have the responsibility of managing cable slack. This job entails pulling cable out of the reel as the crewmember flies away, and then feeding it back into the reel housing as the SAFER returns. Slack must be controlled such that tension is kept to a minimum, yet there is not a risk of entanglement in loose cable. It was unknown if the tension could be kept low enough to avoid affecting SAFER flight control. It was also unclear if the astronaut managing the tether would be able to perceive when the proper amount of slack is achieved. While this might be simple in a $1-G$ environment by observing the sag in a loose cable, zero-G removes this visual cue.

\section{E. Effects of Vehicle Attitude and Orbit}

When the concept of using SAFER for inspection was first conceived, it was recognized that there could be prohibitive flight complexity added depending on the docked Space Shuttle and ISS attitude. The standard attitude for the docked vehicle is a type of Local Vertical Local Horizontal (LVLH) orbit. In this attitude, the orientation of the vehicle with respect to the Earth remains constant throughout the entire orbit.

6

American Institute of Aeronautics and Astronautics 


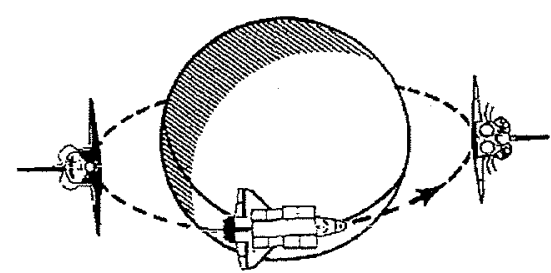

Figure 2. Example of LVLH orbit.

Conversely, the SAFER maintains an inertial orbit because the three internal gyroscopes hold the attitude fixed in space regardless of location in the orbit.

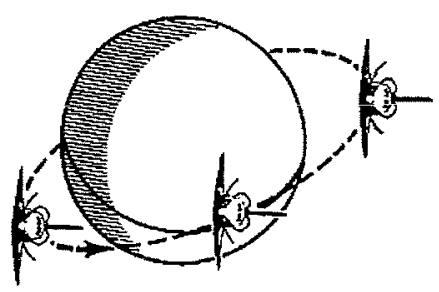

Figure 3. Example of inertial orbit

In very simplistic terms, the difference in orbit types cause the SAFER pilot to perceive that the vehicles rotate 360 degrees over the course of one orbit. Would this relative rotation equate to high propellant consumption as the astronaut attempts to maintain a consistent attitude for inspection? If this action increases SAFER flight difficulty, then the possibility of human error also rises. If so, this may dictate that the Space Shuttle transition to a similar inertial attitude for the duration of SAFER inspection. While this would be possible, it does add mission complexity and undesirably heats localized areas of the ISS thermal control system.

\section{Testing and Analysis}

With crew safety being of primary concern, there was a long road to be traveled before SAFER could be considered an acceptable means of inspection. If any of the problems previously discussed could not be resolved, then a substitute method of Orbiter examination would be required. The conundrum lies in the fact that there is no perfectly analogous test environment on the ground. SAFER performance is dependent upon both zero-G and vacuum. In order to find solutions to all the problems, it was first necessary to identify the critical factors that affect each specific issue. Ground based tests could then be developed which adequately simulate the important elements of each concern. Following is a description of the tests and analysis performed.

\section{A. Risk of Safety Tether Rescue}

Much to the chagrin of the SAFER inspection team, no hardware modifications or elaborate tests will provide a means to violate the laws of physics. Angular momentum will always be conserved and the associated risk of tether retrieval cannot be eliminated. Furthermore, the single string SAFER is not immune to failures which would necessitate a tether rescue. Therefore, no amount of work will ever be able to remove all the risk of an uncontrolled EMU catastrophically colliding with structure. 
In addition to the hardware analysis described previously, the likelihood of pilot error preventing controlled flight back to the robotic arm is controlled by establishing conservative limits on consumables. The Virtual Reality Laboratory was recognized as the only suitable location to evaluate flight skill and consumable expenditure. The simulation models SAFER performance, orbital dynamics, vehicle configuration, and depth perception with excellent accuracy.

\begin{tabular}{|c|c|c|c|c|}
\hline & \multicolumn{4}{|c|}{ Flight Number } \\
\hline & 1 & 2 & 3 & 4 \\
\hline \multirow{4}{*}{ 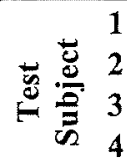 } & $\mathbf{A}$ & A & B & B \\
\hline & B & B & $\mathbf{A}$ & $\mathbf{A}$ \\
\hline & $\mathbf{A}$ & B & B & $\mathbf{A}$ \\
\hline & B & $\mathbf{A}$ & A & B \\
\hline
\end{tabular}

Table 1. Test plan for consumable limit study. Extremely high confidence is needed in any consumable limits that would be set. If one astronaut ever ran out of propellant, a safety tether rescue would be required. This is undesirable considering the danger of relying on the safety tether. Because piloting skills improve with practice and currency, it was necessary to develop a test that removed the effects of learning $\$$. Table 1 shows the two scenario, four period test plan that was chosen for this quality. Without this, the subject would show increasingly efficient skill during repeated simulations. This would in turn bias the results toward a lower propellant and time margin of safety. Eight astronaut test subjects were chosen, each with varying levels of SAFER training, so that every series could be duplicated once.

In this test, scenario A involved having the SAFER crewmember originate at the SRMS and fly to two simulated damage sites. At each location, the crew would achieve a stable hover between 5 and 15 feet away from the tile surface. This distance has previously been identified as the range that provides the desired image resolution for digital pictures with a $50 \mathrm{~mm}$ lens. The pilot would then slowly approach the tile surface and hover within arms length such that the handheld contour gauge could measure depth cross sections. Scenario B was identical to A

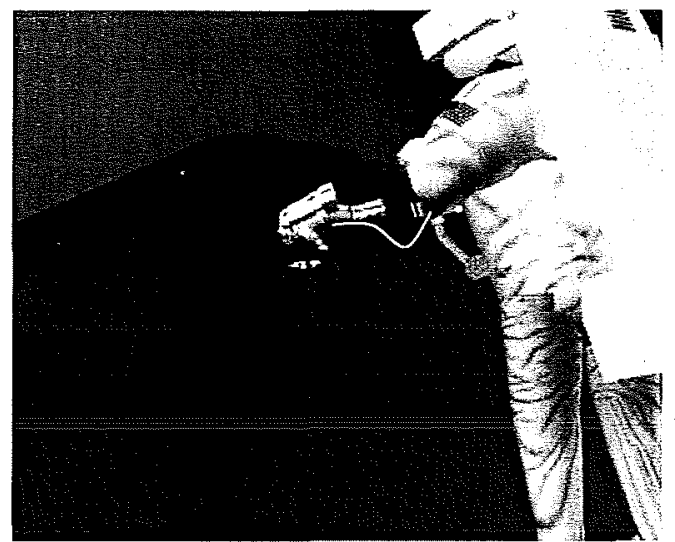

Figure 4. Simulated tile inspection in VR lab. except three inspection locations were visited. If this were performed in flight, the second crewmember on the SRMS would be able to provide additional situational awareness through voice communication. Likewise, while utilizing a simulated view from the second crewmember in the VR lab, the test conductor was able to provide verbal assistance during the test.

For data collection, each scenario was divided into similar tasks. Gross translations included the flight between the SRMS and damage sites, as well as the motion from one damage location to the next. Approach tasks involved the more precise flight from the photography location to the vehicle surface for depth measurement. Thus, scenario A included three gross translations and two approaches whereas scenario B contained four gross translations and three approaches. For each task, propellant consumption and elapsed time was recorded.

The second aspect of risk reduction for tether retrieval involves safe procedure development. Testing this operation proved extremely difficult due to the limitations of the available facilities. The crewmember positioned on the robotic arm needs to tug on the tether cable to pull the stranded pilot to safety. The VR lab cannot model this particular force feedback problem accurately. A preliminary test was conducted on the Precision Air Bearing Floor (PABF). This facility offers virtually friction free motion in one plane by elevating support sleds on a cushion of pressurized air. A tether was connected to a sled with similar mass properties as an EMU and then test subjects pulled the cable. Unfortunately, the PABF test proved to be inconclusive because the floor limits motion to two degrees of freedom. The test was repeated onboard NASA's KC-135 aircraft. This plane flies a series of parabolic trajectories at the top of which twenty seconds of zero-G can be experienced. For this test, a free-floating test subject with an additional 400-pound mass simulator was located on the end of the extended safety tether. A stationary crewmember held the tether reel fixed in place. Subtly different retraction techniques were tested in hope of determining which method provided the greatest control ${ }^{* * *}$. As was expected, there were aspects of the $\mathrm{KC}-135$ test

$\S \S$ Although crewmembers generally receive proficiency SAFER training during the week of quarantine prior to launch, this is not always achievable. In some cases up to a month may have elapsed between the last training and flight. Because of this, the benefits of recent training were not desired for setting consumable limits.

${ }_{* * *}$ Variations included: Fixed crewmember initiating pull, free-float crew initiating pull, controlling trajectory by pulling cable to one side, tether connected at location of free float crew's EMU D-ring or held so tension is directed through center of gravity. 
that did not accurately represent flight. The most significant was the limited size of the cabin. The test subject would hit a wall after floating five feet, whereas an EVA crewmember could swing out an 85-foot arc on orbit.

\section{B. Effects of Vehicle Contact Forces}

There are several complex factors influencing the resultant crewmember motion after contacting the vehicle. This meant that simple conservation of momentum calculations would not suffice for determining flight controllability and propellant consumption. The need to include the effect of the SAFER attitude control system as well as astronaut piloting skill meant that the final evaluation would need to be performed in the Virtual Reality lab. However, it is equally important to include an accurate representation of the contact pressure, duration, and location with respect to the EMU's center of gravity. These are all going to be affected by depth gauge ergonomics, measurement technique and crewmember range of motion in the spacesuit. The VR lab is not an ideal environment for evaluating these aspects.

Two methods were conceived to create a marriage between the virtual reality simulation and measurement reaction forces. The first involved a series of tests on the Precision Air Bearing Floor and onboard the KC-135 aircraft. Crewmembers were suited in an EMU and slowly pushed toward a damaged tile board to simulate SAFER flight approach for measurement. A prototype depth gauge tool was used in which a pair of single axis load cells would continuously monitor the compression force during the measurement process. Figure 5 shows a preliminary version of the depth gauge with the integrated load cells. The total force exerted by the crewmember during depth measurement was obtained by summing the force registered by each load cell. Several plots of force over time were created as crewmembers measured damaged tile with various initial flight velocities.

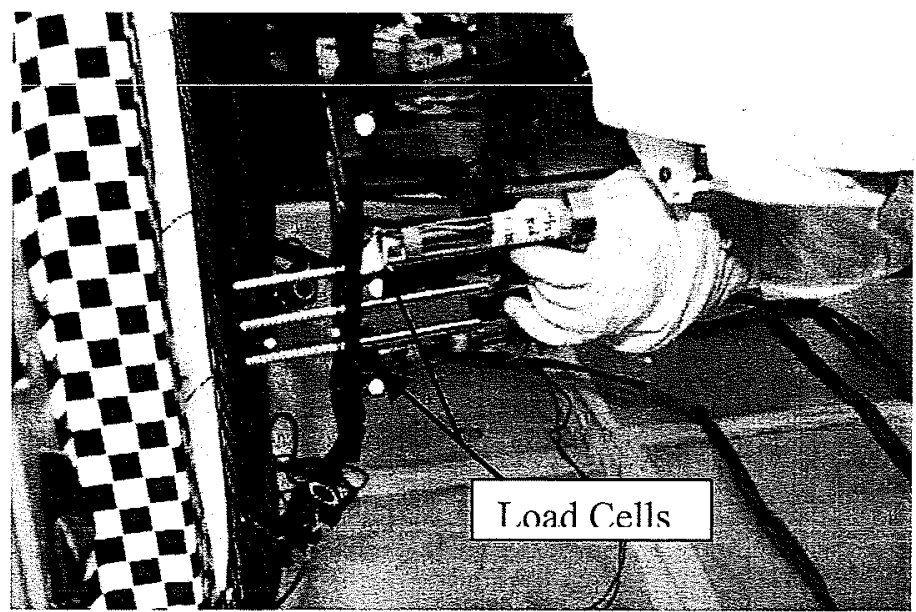

Figure 5. Prototype depth gauge during a test on the KC-135.

The end goal was to take the forces recorded during actual suited depth measurements and include them in the Virtual Reality simulation. When the virtual tool makes contact, the Orbiter would impart the reaction force seen during testing. The force plot corresponding to the approach speed that most closely matched that flown in the simulation could be applied.

The KC-135 aircraft is by nature a dynamic test bed. The most subtle non-zero $\mathrm{G}$ forces and flight turbulence can impart unrealistic motion that can corrupt data. A quantitative means of filtering out the bad data was needed. Hence, the Anthropometry and Biomechanics Facility (ABF) at Johnson Space Center employed a threedimensional, video based, motion analysis system called Vicon. This system was used to capture the movement of the EMU in relation to the damaged tile area thereby, allowing particular test cases most closely representative of flight conditions to be selected. A synchronization signal was used to match the motion analysis data with that from the load cells. This was necessary to differentiate the accelerations caused by tool contact from those due to outside factors such as turbulence or contact with the test director.

The Vicon system includes an array of ten video cameras, each of which is surrounded by LEDs. Light from the LEDs is reflected off markers strategically positioned on the EMU and the tile surface and is then capture by the camera lens. From this signal, the distance from a camera to each marker is determined. The position in threedimensional space is calculated by triangulating the information from all ten cameras. By applying calibration and reconstruction algorithms, a digital model of the spacesuit motion is created. Marker positioning was chosen to focus on the location of the depth gauge, helmet, boots, and center of gravity (CG). Figure 6 shows the test setup on the $\mathrm{KC}-135$. The following information was obtained during each test case.

- EMU CG trajectory through the $X-Y$ and $X-Z$ planes

- EMU CG linear speed

- EMU pitch and yaw angle with respect to tile surface

- EMU angular velocity about the yaw and pitch axes 


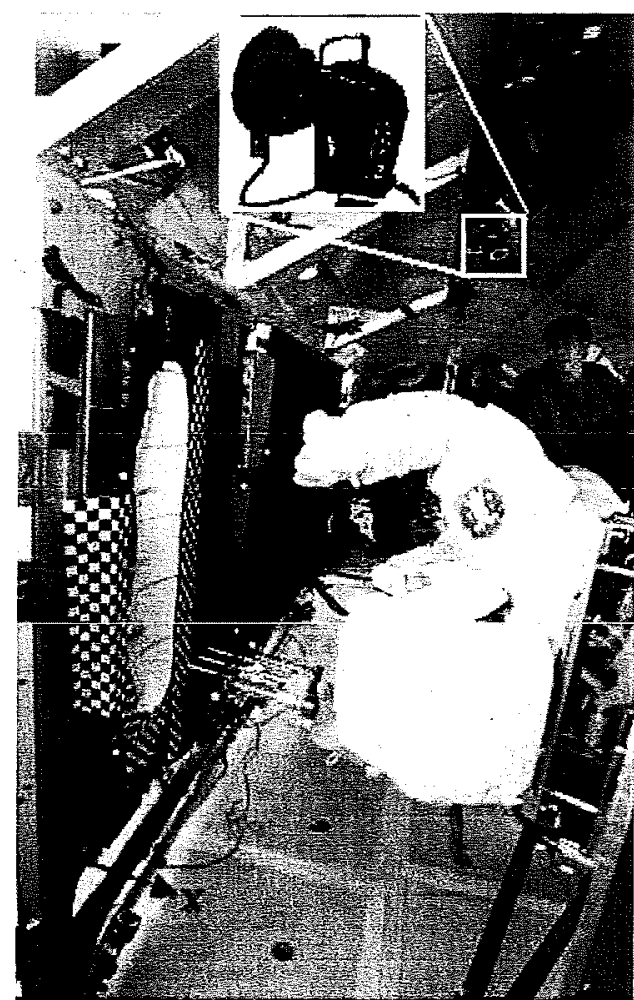

Figure 6. KC-135 test configuration.
The motion analysis combined with standard video footage filled another critical role in the overall test integration. After including the force data in the virtual reality laboratory, the simulation was tested and the resultant motion was compared to that seen on the KC-135. Without the influence of SAFER's attitude control in the aircraft tests, rotation caused by the contact would be greater than that in the VR lab. Similarly, the magnitude of the linear accelerations should be approximately equal in both locations. If major differences were observed in the VR simulation, this would indicate that sources of error were large enough to affect the model accuracy ${ }^{\dagger \dagger}$. If this approach to upgrading the VR simulation created unrealistic motion during tile contact, it would need to be abandoned in favor an alternative.

The second method that was developed for modeling the effects of vehicle contact utilized a robotic force-feedback system in the Virtual Reality Lab. Previously this tool was used to simulate the dynamics of large mass handling during EVAs. The robot is named Charlotte because it is suspended by a spider-like web of eight cables, each connected to a motorcontroller. Four sensors on Charlotte measure torque and force applied by a crewmember. The output of these sensors is fed into a simulation that then drives the motors to adjust the cable length and move the robot. The model can be modified so that the mass properties of the object being manipulated can be controlled. Thus, if Charlotte were used to represent a massive object, a crewmember would find it harder to move than a lighter one due to the high inertia.

Adapting Charlotte for use during SAFER flight had not been attempted previously. The robot was made to represent an area of the Space Shuttle's tile surface. To aid in visualization, a photograph of damaged tile taken after the STS-27 mission was mounted on Charlotte's front surface. During SAFER flight in the VR lab, the astronaut is constrained to sit in a chair. Rather than the EMU being pushed away from a stationary Orbiter, the model had to be built so that the vehicle moved away from the motionless pilot. This is acceptable because the relative motion between the two is the same either way. If these modifications were made successfully to the simulation, Charlotte would physically move toward the crewmember as they flew SAFER towards the virtual vehicle. When the astronaut reached out their hand to take a depth measurement, Charlotte would sense the contact and react accordingly.

\section{Body and Tool Position Awareness}

A limitation of the VR lab is that the SAFER pilot cannot get a representative understanding of their body position. While wearing shirtsleeves, it is impossible to make any conclusions about how well one would perceive their EMU's proximity to the shuttle. This can only be determined while inside an EMU. Suited evaluations are commonly conducted in the Neutral Buoyancy Laboratory (NBL). The underwater environment is the best location for simulating prolonged weightlessness for EVA training and tests. Support divers held two astronauts, outfitted with the complement of tools needed for inspection, next to a simulated tile surface. The crewmembers' bodies were then pitched through a range of angles with respect to the tile until acceptable body positions could be enveloped. If the pitch angle was too low, the crew became concerned about accidental boot and tool contact. At high pitch angles, helmet contact and operation of the contour gauge became the concern. Although this was useful for determining the rough range of acceptable positions, the information could not be depended upon. In the NBL, proper buoyancy is achieved by attaching leg weights to the spacesuit. This has the effect of pulling the boots towards the pool floor and straightening out the legs. In addition, the training suits are not as stiff as the flight units due to the frequency of use and constant exposure to water. The consequence of these differences is that the knees and boots do not extend as far in front of the astronaut in the NBL. By reviewing previous flight photos, it was

t†† Possible sources of error include load cell accuracy, aircraft accelerations, and differences between simulated and flown EMU mass properties. 
possible to determine the EMU's neutral joint angles. A comparison of NBL and flight leg positions is shown in Fig. 6.

The challenge now became how to constrain the leg joint angles to match flight positions. This objective was captured during the dry run test of the Vicon motion analysis system on the Precision Air Bearing Floor (PABF). Because body pitch is the most relevant in selecting an attitude for measurement, a pair of sleds was built to hold an EMU on its side. This provided unencumbered motion about the pitch axis. The two sleds constrained the EMU legs such that the joint angles matched those seen on orbit. Again, the crewmembers were positioned in front of a tile board at various angles until they could determine their comfort zone. An overhead camera was used to measure the pitch angle once the preference was formed. In addition,

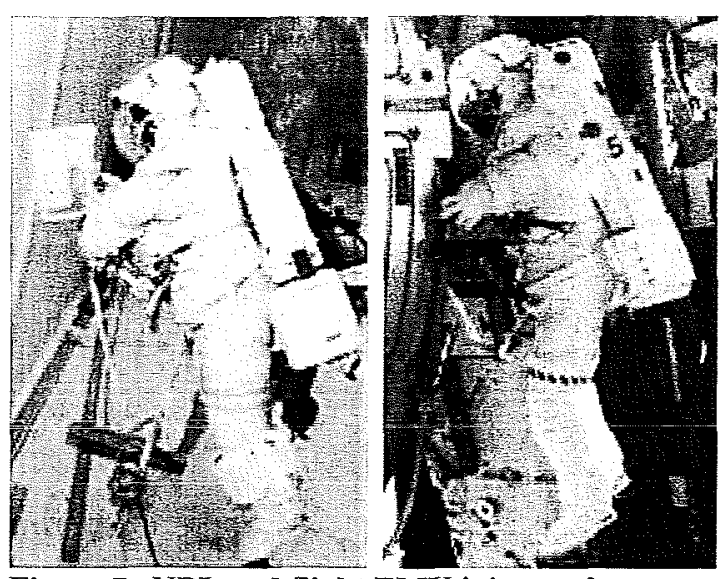

Figure 7. NBL and flight EMU joint angles

a load cell was located behind the tile board to record the magnitude of any inadvertent contact that might occur during damage inspection. If it turned out that accidental contact was unavoidable, this force data would provide a means for determining if tiles would be damaged.

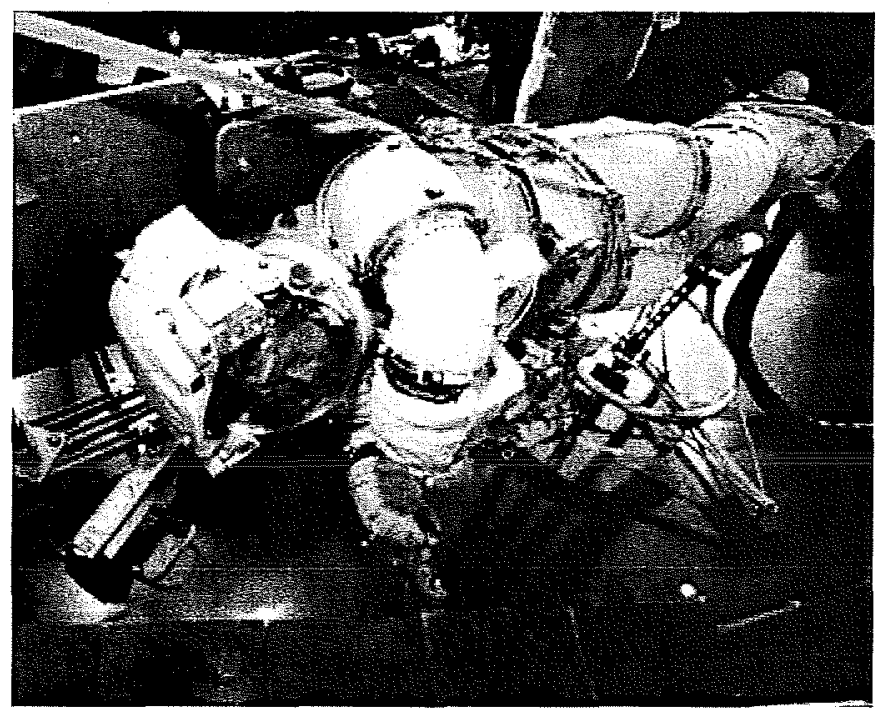

Figure 8. EMU secured on sleds during PABF testing.

\section{Safety Tether Slack Management}

Safety tether slack control is necessary for efficient SAFER flight. Any nitrogen propellant that is spent counteracting the cable tension becomes unavailable as safety reserves. A test is needed to determine how well a crewmember can control the safety tether cable with cumbersome EMU gloves. All 1-G tests are invalid due to the added tension caused by cable sag. In addition, it is equally important to find out how well the crewmember can perceive that the cable is slack enough to avoid pulling on SAFER, yet controlled enough to prevent entanglement. Unlike zero-G, gravity creates a consistent bow in the tether that invalidates any tests that attempt to model the visual cues.

The necessity of a zero-G environment meant that the $\mathrm{KC}-135$ aircraft was the only adequate testing facility. One test subject representing the crewmember in the arm's foot restraint stood in a fixed location. This person wore EMU gloves while extending cable from the tether reel as needed. A second subject representing SAFER, floated down the cabin while holding a force gauge in-line with the cable end. The SAFER subject limited their speed to less than one $\mathrm{ft} / \mathrm{s}$. While this speed is actually orders of magnitude faster than that planned for flight operations, the difference will only add conservatism to the results. This is because the faster speed during testing makes it harder to control the cable and is more likely to induce tension; flight forces would be more benign. During the tether 
extension, average tension was recorded as well as the peak force. Although it was expected that the tension would be trivial, if significant forces were measured they could be incorporated into the virtual reality simulation for further study of propellant impacts.

\section{E. Effects of Vehicle Attitude and Orbit}

As has been discussed, an EVA crewmember will face the challenge of maintaining a consistent attitude with respect to the Orbiter. This is crucial for avoiding unwanted contact with TPS material. The virtual reality laboratory was chosen to test what effect the Shuttle and ISS attitude have on the SAFER inspection difficulty. Because SAFER uses an inertial attitude hold, it was expected that frequent yaw and pitch corrections would be necessary when the vehicles were flying an LVLH attitude. The consequences of the additional corrections had an unknown effect on SAFER consumables.

A VR lab test was developed which compared inspection missions with the vehicles in both LVLH and inertial orbits. For the both cases, standard docked vehicle attitudes were used. Six subjects performed the inspection cases as described in table 2.

During the test, propellant consumption and elapsed time for each stage of flight was recorded. In addition, subjects were asked to describe their perception of the flights so that a qualitative comparison of the difficulty could be understood.

\begin{tabular}{|c|c|c|}
\hline & $\begin{array}{c}\text { Number of } \\
\text { Damage } \\
\text { Sites Visited }\end{array}$ & $\begin{array}{c}\text { Vehicle } \\
\text { Attitude }\end{array}$ \\
\hline Case 1 & 2 & Inertial \\
\hline Case 2 & 2 & LVLH \\
\hline Case 3 & 3 & Inertial \\
\hline Case 4 & 3 & LVLH \\
\hline
\end{tabular}

Table 2. Orbit tvpe impact test plan.

\section{Results}

Like the slow assembly of a jigsaw puzzle, each test added a small piece in the overall understanding of the big picture. At any point, a single negative result could have threatened the project and left the Space Shuttle Program without the option of using SAFER for vehicle inspection. The test results for each issue are summarized here.

\section{A. Risk of Safety Tether Rescue}

The purpose of the different consumable limits was the key factor in deciding amount of statistical confidence desired. SAFER control may be lost if the propellant is consumed or if the unit is powered on for more than fortyfive minutes. The paramount concern is to preserve ample nitrogen and operational time so that the astronaut can fly back to the safety of the robotic arm. This task is the same as one gross translation performed in the VR lab tests. Because it is so important that this phase of the flight is successful, $99.9 \%$ confidence was chosen for establishing the limits. Statistically this means that pilot error will only necessitate a safety tether rescue once in a thousand inspections. The desired level of confidence is achieved by reserving $22 \%$ of the nitrogen and 13 minutes for the return flight. However, thruster performance is degraded when the propellant tank approaches empty. Thus, it was decided that the SAFER pilot shall discontinue all inspection work and fly back to the arm if the remaining propellant reaches $30 \%$.

Space Shuttle flight history has seen as many as 298 dings on tile that were larger than one inch. It stands to reason that future missions may require detailed inspection of multiple locations. A second limit must be established for these situations. After completing a damage inspection, the astronaut must decide if they should return to the arm or proceed to the next examination site. The remaining propellant may be above $30 \%$, however it would be foolish to attempt another inspection if it were insufficient to complete the task before reaching this limit. Before committing to this undertaking, the crewmember must be reasonably sure that they have time and propellant suitable for completing the job. Additional inspections require two gross translations and one approach to complete the task and return to the arm. Based on the VR tests, this can be achieved with $99.0 \%$ confidence when at least $45 \%$ nitrogen remains and when the elapsed time since powering on SAFER is less than 23 minutes. Even if the decision is made to proceed to another location, that inspection will be aborted if the nitrogen level drops below $30 \%$ or the elapsed time is greater than 32 minutes.

It is important to recognize some factors that affect the accuracy of these results. First, the crewmembers that were selected as test subjects were only required to have generic SAFER training. Flight assigned crews will receive significantly more training to prepare them for potential SAFER TPS inspections. A second factor is that test development for all of the problems covered was happening in a parallel timeframe. The implementation of Charlotte to model vehicle contact forces was not complete until after all consumable testing was complete. This creates a low bias on the propellant used during the approach flight segment. This is considered acceptable because the $30 \%$ limit that protects crew safety is not based upon the approach data. The worst-case effect of this uncertainty is that it may not be possible to inspect as many damage locations as previously believed. 
The unavoidable shortcomings of the PABF and $\mathrm{KC}-135$ made tether retrieval tests mostly inconclusive. However, there were some useful lessons to be gleaned from the KC-135 flights.

1) To reduce uncontrolled spinning, the safety tether cable should be connected to the EMU D-ring on the side that will be kept closest to the robotic arm. When tension is applied to the cable, the suit is in a stable equilibrium, which reduces rotation. If the cable were connected to the opposite side of the EMU, a tug would spin the suit as the far connection point leads the motion.

2) Before the EVA crewmembers begin pulling the tether, the SAFER pilot should fairlead the cable through a glove positioned in front of their chest. This action directs the pull through the EMU's center of gravity. Positioned here, moment arms are minimized and unwanted spin is reduced. This also prepares the crewmember to counteract rotation and control their attitude by diverting the cable to either side. A clockwise spin can be overcome by holding the cable to one's right side. Likewise counterclockwise rotation is corrected with the cable diverted to the left. It is noteworthy that this technique is less effective at controlling pitch and roll because there is a greater moment of inertia about these body axes. Care must also be taken to avoid an excessive increase in the linear velocity of the crewmember with each pull.

Without a testing environment that is sufficiently analogous to space flight, procedure development was forced to be somewhat of an unverified mental exercise. The EMU has been certified to withstand impacts at speeds less than $1.6 \mathrm{ft} / \mathrm{s}$. Even if SAFER failed such that thrusters fired when uncommanded, the crewmember can detect this anomaly and disable the jets before reaching a speed of $0.5 \mathrm{ft} / \mathrm{s}$. Even so, by the time the EMU is pulled within reach of the second crewmember it would have accelerated to over $10 \mathrm{ft} / \mathrm{s}$ in the worst-case. The only relief to this problem can be seen by re-examining Eq. 3 .

$$
R_{0} V_{0} \sin \theta_{0}=R_{I} V_{I} \sin \theta_{I}
$$

The magnitude of $R$ decreases when the EMU moves closer to the robotic arm. The initial velocity, $V_{0}$ must be minimized to prevent excessive speeds during the rescue. This can only be achieved with controlled impacts with structure prior pulling the stranded crewmember closer to the robotic arm. Using the cable manipulation techniques described above, the SAFER pilot should attempt to orient their body so that they are facing the structure during contact. If successful, the crewmember's hands could dampen the impact and reduce $V_{0}$. The crew may even be able to hold onto the vehicle depending upon where the impact happens. There are a couple of key points to remember if this procedure is needed.

1) The astronaut that is tending the tether from the robotic arm should apply the necessary tension to prevent the SAFER crewmember from drifting farther away. Contrary to common instinct however, the tether should not be retracted. This will cause the stranded EVA crewmember to swing out an arc that will eventually bring them to structure. The resultant contact will provide a place to hold, or can be used to reduce the angular momentum so that a subsequent cable retraction can be performed.

2) Controlling the initial velocity at the time of SAFER failure is critical. SAFER training should emphasize slow movement and rapid recognition of thruster failures.

\section{B. Effects of Vehicle Contact Forces}

The tests performed on the KC-135 to capture motion analysis and load cell data were prone to unavoidable sources of error, which made it extremely difficult to capture quality data. Despite having performed a dry run of the Vicon system on the PABF, three flights worth of motion analysis data was unusable. From the three remaining flights, data from only two parabolas were identified as candidates for incorporation in the VR model. Several factors listed below were considered in the data reduction process.

1) The test conductor must not be touching the subject for a period before, during, and after contour gauge contact with tile.

2) The EMU should not exhibit linear or rotational accelerations when not in contact with anything. This would be an indication of aircraft turbulence.

3) The EMU's center of gravity must be moving at a constant speed less than $0.2 \mathrm{ft} / \mathrm{s}$ toward the tile with no rotation prior to tool contact. After depth measurement, the EMU can no longer be moving toward the tile board.

4) The body attitude must have a pitch between 20 and 40 degrees with negligible yaw.

5) Video footage must show that the depth gauge was the only item touching tile. Contact with a second hand, helmet or boot would impart a load not recorded by the force sensors. 
Figures nine and ten show a selection of the motion analysis data set from the test case chosen for use in the Virtual Reality model.

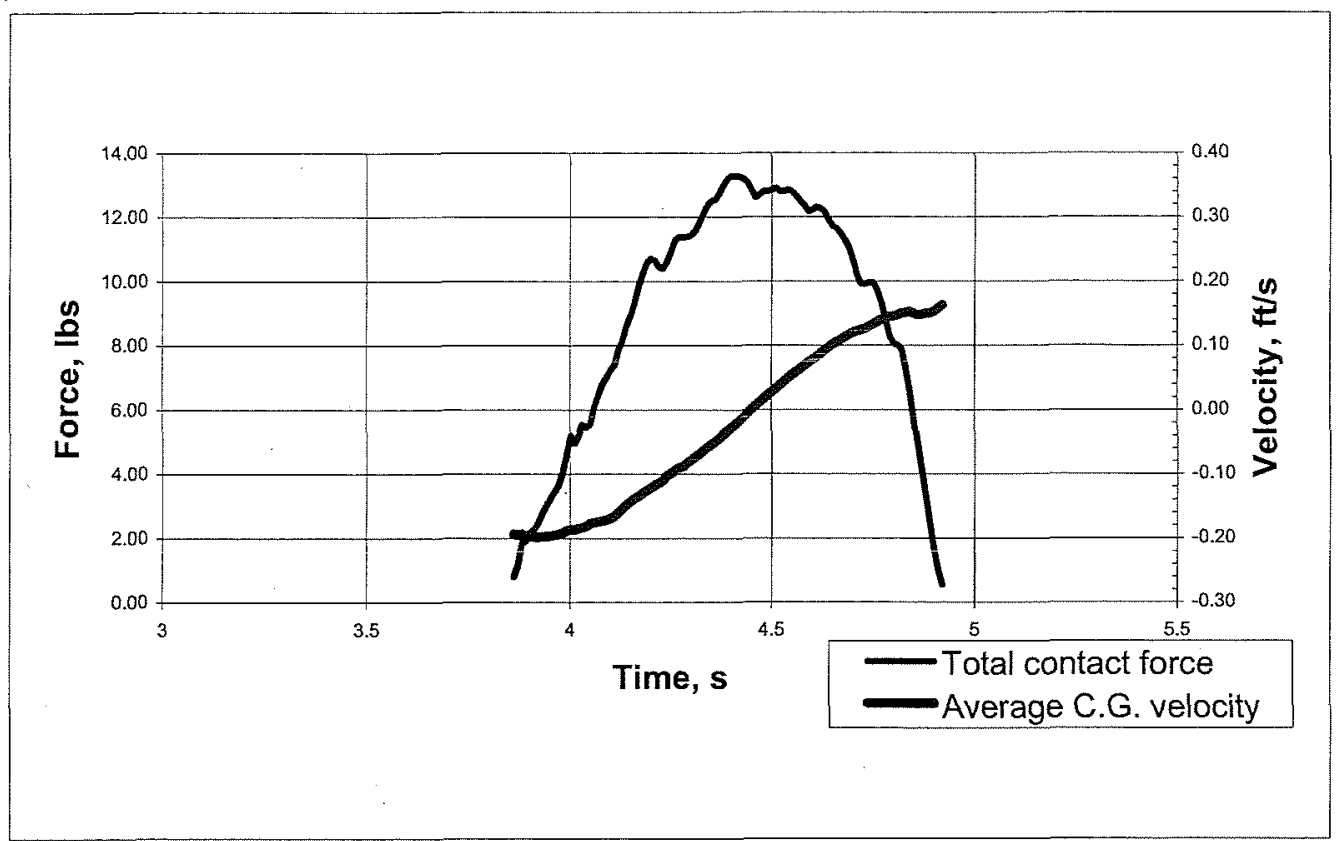

Figure 9. C.G. velocity and tool contact force during $\mathrm{KC}-135$ depth measurement.

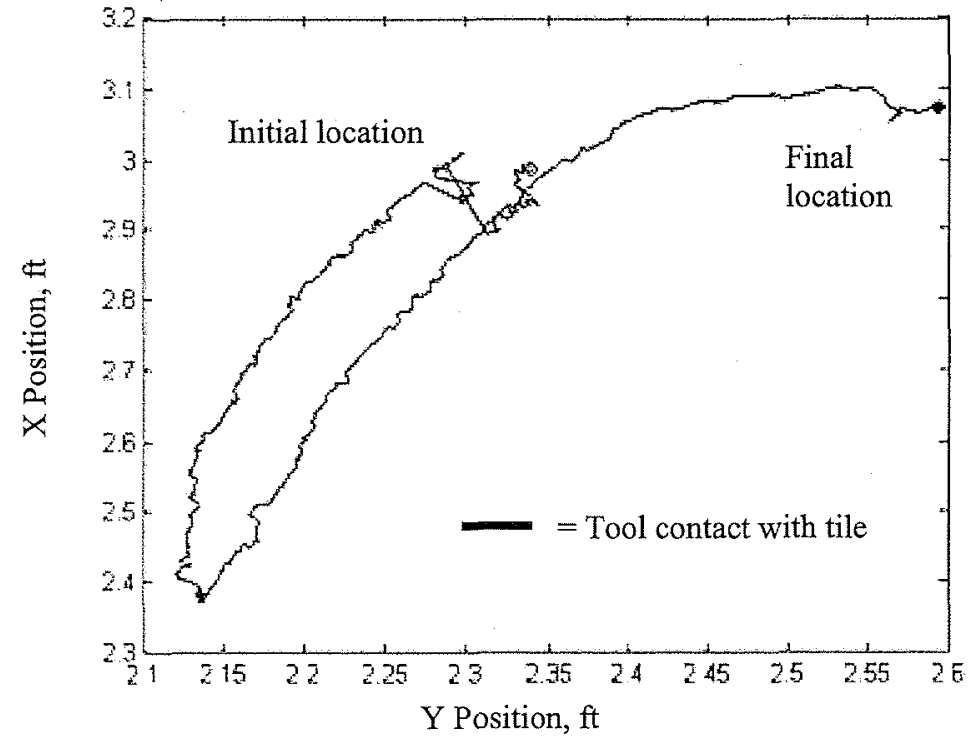

Figure 10. C.G. trajectory in the test $X-Y$ plane

Unfortunately, when the VR simulation was tested with the force data incorporated into the model, the result was not consistent with what was observed during the $\mathrm{KC}-135$ flights. The simulated contact created unrealistically high linear and rotational accelerations. A basic conservation of momentum calculation was performed to determine if the problem lied in the simulation, or the force data being used as an input. This calculation showed that the physics driving the VR model were correct and the problem was resulting from the force data. Most likely, the measured loads were unrealistically high due to sensor bias, or non-zero $G$ forces induced by the aircraft. At any rate, the development of Charlotte was nearing completion and that system was showing promise. Any attempt to correct the problems with the $\mathrm{KC}-135$ force data would be put on hold pending the outcome of the Charlotte trial tests. 
Incorporating Charlotte into the SAFER flight simulation was amazingly successful. The simulated EMU reaction to depth measurement was analogous that seen on the $\mathrm{KC}-135$. The major difference was that the SAFER attitude control was now able to affect the flight dynamics. The updated virtual reality model shows that EMU rotation resulting from a typical measurement is stabilized by SAFER before large angular displacements occur. The motion is not severe enough to cause crewmember disorientation. Despite this, Charlotte has shown that every effort must be made to minimize contact force. The propellant consumed to counteract contact is not trivial over the course of an inspection mission to multiple damage locations. Excessively strong measurements will result in loss of controlled flight. Unlike the prerecorded force plots, Charlotte will teach astronauts the effect of their specific measurement technique. This will be instrumental in sensitizing astronauts in the need for a light touch.

\section{Body and Tool Position Awareness}

The air-bearing floor testing that was performed to develop a body position during damage depth measurement revealed a surprising result; all astronaut test subjects independently arrived at the same answer. The unanimous finding was to position the crewmember such that the angle between the long axis of the EMU's life support system backpack and the tile surface is between 20 and 40 degrees. Although the crewmembers reported that they could not see their boots, they were confident that there was enough clearance. An overhead camera and the tile board load cell both confirmed that there was no accidental tile contact. These findings were later confirmed during the motion analysis flights onboard the $\mathrm{KC}-135$.

In addition to establishing preferences for body positions, configuration of EVA tools proved equally important. The SAFER hand control module protrudes in front of the crewmember. This prevents the mini-workstation T-bar ${ }^{\text {fł }}$ from being fully stowed. If placed in the most upward achievable position, the T-bar will obstruct short-armed crewmembers from reaching the tile with the contour gauge. In addition, this T-bar configuration increases the chance of inadvertent contact with TPS materials. Therefore, procedures have been written to completely fold the Tbar down to avoid these interference issues during inspection. It was found to be wise to minimize the number of tools taken to the inspection worksite. This makes one-handed operation easier, and generally simplifies the task. It is important to note that the tiles were also tested to determine the extent to which they can withstand accidental EVA contact. An EMU boot kick test revealed that tile damage, while possible, was only superficial. Regardless, it seems sensible to minimize all unnecessary contact.

\section{Safety Tether Slack Management}

Fortunately, the results for the slack management tests proved very favorable. All crewmembers involved with the test agreed that it was a simple job. The average running tension on the cable varied between 0.0 and $0.1 \mathrm{lbs}$. Peak readings were generally about $1 \mathrm{lb}$. However, the personnel conducting the test were able to feel when the peak forces occurred and reported that they were very infrequent with durations less than approximately one quarter second. These data indicated that tether tensions were managed to a negligible level. Because of this, it was decided to not include tether forces in the virtual reality simulation model.

\section{E. Effects of Vehicle Attitude and Orbit}

Unlike the other problems addressed so far, the concern over flying SAFER in close proximity to a Space Shuttle in an LVLH orbit never jeopardized the feasibility of the project. If the LVLH vehicle attitude were found to be unacceptable, it would be possible to impose attitude restrictions on ISS and the Orbiter during SAFER inspection. However, these restrictions are certainly undesirable due to the effects on ISS systems. As it turned out, the comparative virtual reality tests showed that there was no significant difference between inspections flown with the vehicles in LVLH or inertial orbits. On average, the LVLH test cases used $1.4 \%$ more nitrogen during gross translations, but $1.3 \%$ less during approaches. The average duration of LVLH missions was only 59 seconds longer over the course of a 22-minute inspection. These difference are considered insignificant and well within the margin of error for the test. Additionally, all crewmembers involved with the test agreed that either situation was acceptable. These results provide operations flexibility by allowing any vehicle attitude to be flown throughout SAFER flight.

Although the study proved that the orbit types did not affect consumables or flight duration, the test did verify important operational impacts that must be addressed during crew training. As previously discussed, the LVLH orbit causes the vehicle to rotate 360 degrees with respect to SAFER over the course of each orbit. This equates to as much as 180 degrees of relative rotation during an inspection mission. Unlike SAFER rescue, the close proximity to structure inherent in TPS examinations makes this effect relevant. The SAFER pilot needs to be vigilant to correct

¥łł The mini-workstation T-bar is a device used to stow multiple EVA tools near the astronaut's chest for easy access. The T-bar pitch can be adjusted 135-degrees.

15

American Institute of Aeronautics and Astronautics 
attitude deviations. The support EVA crewmember on the robotic arm must also provide verbal assistance to make the pilot aware of slow changes in body orientation. A final caution must be expressed regarding the applicability of these results. The vehicle attitudes chosen for this test are common for docked phases of flight. However, the conclusions may not be valid for all possible attitudes. If a SAFER inspection is ever performed, simulations should be repeated with the flight specific attitude and damage locations to ensure that the task is still acceptable.

\section{Conclusion}

As we looked to the East Texas sky on a Saturday morning in February, the arms, which held high a banner displaying the mantra "Failure is not an option," began to quiver under the burden of reality. From our loss is born a renewed sense of purpose. The previous success that had come to be considered routine would never again be taken for granted. To continue the legacy of human space flight, we must learn from previous mistakes to ensure they are never repeated.

The loss of Columbia and her crew has demonstrated the vulnerability of the Space Shuttle's Thermal Protection System. Inspection capability must exist to verify its integrity after the chaotic launch into orbit. Furthermore, backup options must be ready in the event that performance of the primary inspection tools fall short of expectations. If we have done our job correctly, SAFER will never be used for inspection. However, preparation for contingencies dictates that all obstacles facing the use of SAFER for TPS inspection are investigated to provide this capability.

If failures or other considerations lead to the election of SAFER inspection, the testing and analysis results reveal the task to be within the hardware and crew's capability. Every EVA has operational impacts and hazards. Therefore, autonomous and robotic methods should always be preferred over any EVA option. Of the manual inspection alternatives, those that provide the greatest stability at the worksite and ease of emergency retreat should take precedence. Operational overhead and mission impacts should also be considered for each specific flight. In certain situations, SAFER flight will be the best plan, and the operation can be considered sound.

It should be understood clearly that SAFER inspection is not without risk to the Orbiter and EVA crew. The greatest concern is with safety tether retrieval. Great strides have been taken to increase operational reliability thereby reducing the chance of performing this procedure. If a tether rescue is conducted, it will be complex and the stranded crew will have little body control. However, procedures have been developed to give the crew every possible advantage to affect a successful rescue. It should be remembered that this is not a new risk; every EVA is exposed to similar dangers when using a safety tether. As has already happened at least three times on orbit, when a crewmember loses grasp on a handrail they are dependent upon the safety tether to return them to structure. Such an event could easily swing the crew into TPS materials or other equally hazardous areas. One might argue that a tether retrieval during SAFER flight is actually less of a risk because the second EV crewmember is prepared to manage the extended cable length.

Human factors play a paramount role in piloting performance. Compared to SAFER rescue flying, the inspection tasks are made much easier due to the slow translation speeds and close proximity to structure. The short-range flights provide excellent depth perception and relative motion cues. In addition, the added situational awareness provided by the second crewmember results in a new level of flight precision.

A final remark should be voiced on the conclusions drawn in this report. This study is based on the concept for vehicle inspection and repair as of September 2004. Over time, operational priorities and risk tolerance may change, which may draw into question the acceptability of SAFER inspection. Accordingly, the issues presented should be readdressed when this happens to make sure old assumptions are still valid. 


\section{Afterward}

In the time since the original drafting of this paper there has been a significant breakthrough in EVA TPS inspection. Through the use of an imagery analysis technique called photogrammetry, 3D depth measurements can be acquired with the digital camera alone. This eliminates the need to contact the vehicle surface during the inspection process. SAFER flight is much more stable because there is no longer any reaction force to counteract. Propellant consumption is appreciably reduced along with flight duration. Precise body position is no longer as critical because the crewmember can remain over five feet away from the vehicle at all times. There is no risk of further damage to the delicate TPS materials. Tool management is simplified because the contour gauge is not carried to the worksite. This reduces pilot distraction as well as tether entanglement. Finally, the data quality far exceeds that which is possible with the contour gauge.

Photogrammetry has a long history of use in commercial applications. It is used to verify 'as built' dimensions of manufactured parts. The USGS uses it to create topographical maps of the Earth. NASA has even used photogrammetry to measure solar array deflection on ISS and to map the lunar surface. This extensive heritage means that the analysis methods and image requirements are well understood. The analysis relies on photographs taken from slightly different locations. The shift in perspective is used to perform analytical measurements accurate to with $+/-0.05$ inches. In much the same way as the old toy stereo-viewers created the perception of a 3D object, images of tile damage can be combined such that a cavity can be perceived to have depth when a special pair of glasses is donned. $\mathrm{CAD}$ models can be generated, and rapid prototypes of the damage tile can be recreated for use in Mission Control. Most importantly, the Space Shuttle Damage Assessment Team can get an accurate understanding of tile damage regardless of the OBSS functionality. 


\section{Acknowledgments}

Z. C. Scoville and S. Rajula would like to thank the following individuals for their help on the SAFER Inspection project: Jeff Hoblit, Ken Huffman, Brad Bell, David Homan, Ernest Bell, Clark Thompson, Curt Carlton, Dana Weigel, Dina Barclay, Richard Hackett, Nancy Patrick, Rob Boyle, Kevin Lewis, John Chladek, Ronald Cook, Laura Adair, Nick Grigordiadis, Christopher Paul, Paul Dumm, John Michael Tucker, Sarah Skrobarcyzk, Daniel Nguyen, Robert Sweet, Cuong Nguyen, Eric Darcey, Bill Lee, John Harvey, Christopher Prouty, Mark Dub, Donn Liddle, Soichi Noguchi, Steve Robinson, Scott Parazynski and many others.

\section{References}

${ }^{1}$ Columbia Accident Investigation Board, "Columbia Accident Investigation Board Report Volume 1," August, 2003 page 174. 